\title{
Domains of the Pavarotti kinesin-like protein that direct its subcellular distribution: effects of mis- localisation on the tubulin and actin cytoskeleton during Drosophila oogenesis
}

\author{
Gianluca Minestrini, Endre Máthé and David M. Glover* \\ Cancer Research Campaign Cell Cycle Genetics Group, University of Cambridge, Department of Genetics, Downing Street, Cambridge CB2 3EH, \\ UK \\ *Author for correspondence (e-mail: dmg25@mole.bio.cam.ac.uk)
}

Accepted 12 November 2001

Journal of Cell Science 115, 725-736 (2002) (C) The Company of Biologists Ltd

\begin{abstract}
Summary
The kinesin-like protein encoded by pavarotti (Pav-KLP) is essential for cytokinesis and associates with the central part of the late mitotic spindle and interphase nuclei in somatic cells (Adams et al., 1988). Here we define regions of the molecule that regulate its subcellular localisation and study the consequences of overexpressing mutant forms of the protein during oogenesis in Drosophila. Pav-KLP normally associates with the oocyte nucleus, but when over-expressed at moderate levels, its GFP tagged form also accumulates in nurse cell nuclei. At high expression levels this leads to loss of the microfilaments that tether these nuclei, so that they block the ring canals and prevent the 'dumping' of nurse cell cytoplasm into the oocyte, which results in sterility. Localisation to these nuclei is prevented by mutations in either the conserved ATP-binding site of the motor domain or the nuclear localisation sequences in the C-terminal domain. Both such mutations lead to the formation of stable arrays of cytoplasmic microtubules and
\end{abstract}

the progressive disruption of the actin cytoskeleton. The latter is evident by a breakdown of the cortical actin causing disruption of cell membranes; this breakdown ultimately results in the accumulation of cytoplasmic aggregates containing tubulin, actin and at least some of their binding proteins. Pav-KLP is also found associated with the ring canals, actin-rich structures built from remnants of the cytokinesis ring. The stalk domain alone is sufficient for the exclusive association of Pav-KLP to these structures, and this has no consequences for fertility. We discuss whether disruption of actin structures by fulllength cytoplasmic forms of Pav-KLP is a consequence of the resulting stabilised cytoplasmic microtubules per se or accumulation of the motor protein at ectopic cortical sites to sequester molecules that regulate actin behaviour.

Key words: Pavarotti, MKLP-1, Actinomyosin, Drosophila oogenesis, Contractile rings

\section{Introduction}

The kinesin like protein encoded by pavarotti (Pav-KLP) is a member of the MKLP-1 subfamily that includes CHO1 (Sellitto and Kuriyama, 1988), MKLP-1 (Nislow et al., 1992), ZEN-4 (Raich et al., 1998) and KRP 110 (Chui et al., 2000). Mutations in the Drosophila gene pavarotti result in a failure of cytokinesis in cycle 16 of embryogenesis in which mitotic cells fail to form a correctly organised central spindle and never form a contractile ring (Adams et al., 1998). The localisation of the MKLP-1 family members at the central spindle during late anaphase and telophase is consistent with their function in cytokinesis, and once this role is completed these KLPs locate to the mid-body. New protein synthesised during interphase accumulates in the nucleus prior to mitosis. zen-4 mutants in $C$. elegans also show cytokinesis defects (Raich et al., 1998; Powers et al., 1998). Both the B-type aurora kinase (AIR-2) and a GTPase-activating protein for Rho family GTPases (CYK-4) are required to recruit the ZEN-4 protein to the central region of the late mitotic spindle (Jantsch-Plunger et al., 2000; Severson et al., 2000). Members of the Polo protein kinase family have also been implicated in this process as they appear to associate with members of the MKLP-1 subfamily of kinesin-like proteins in mammals and Drosophila (Adams et al., 1998; Lee et al., 1995). Moreover, Pav-KLP and Polo are both delocalised in pav mutant embryos (Adams et al., 1998), and hypomorphic alleles of Drosophila polo show a number of cytokinesis defects in spermatogenesis (Carmena et al., 1998).

A characteristic of gametogenesis in Drosophila is that sperm and the oocyte develop within a cyst of cells. These are derived from a cystoblast that undergoes four rounds of mitosis with incomplete cytokinesis to produce 16 cells interconnected through 15 cytoplasmic bridges that pass through ring canals. The ring canals are built around the remnants of the cleavage furrow and contain several of its molecular components. PavKLP is one such component and is retained in the ring canals of spermatocytes (Carmena et al., 1998). In oogenesis, two of the 16 cells of the egg develop the potential to become oocytes, but then one of these cells loses its synaptonemal complexes and the other adopts the oocyte fate (Carpenter, 1975; Huynh and St Johnston, 2000; Koch et al., 1976). The remaining 15 cells become the highly polyploid nurse cells that are synthetically active and contribute cytoplasm to the oocyte (Verheyen and Cooley, 1994). Oocyte growth begins slowly 
during stages 7-10 and then accelerates during stages $10 \mathrm{~B}$ and 11 owing to fast transport of nurse cell cytoplasm through the ring canals accompanied by regression of the nurse cells. The distribution of Pav-KLP in the early stages of oogenesis had not previously been examined, although the motor protein was described to be associated with the central pole body of the tandem second meiotic spindles (Riparbelli et al., 2000).

Microtubules are required both for oocyte specification and for the accumulation of specific mRNAs in the oocyte at early stages (reviewed in Cooley and Theurkauf, 1994; Theurkauf, 1994). A posterior microtubule organising centre persists from stage 1 to 6 but is lost during stages 7 and 8 (Theurkauf et al., 1992) and is required to establish an anterior-posterior microtubule gradient for asymmetric localisation of axis determinants in the oocyte (reviewed in St Johnston, 1995). Three distinct populations of microtubules coexist within the nurse cell cytoplasm: one associated with the surface of polyploid nuclei; a second extends from the ring canal junctions; and a third dispersed throughout the cytoplasm (Grieder et al., 2000).

The outer rims of the ovarian ring canals, derived from the arrested cleavage furrow, contain F-actin, the tyrosine kinase Tec29 and in younger egg chambers also anillin (Cooley, 1998; Robinson et al., 1994). The inner rim also contains the Hu-li tai shao, Kelch and filamin proteins (Li et al., 1999; Robinson et al., 1994). In addition to disrupting the inner rim of ring canals, mutations in cheerio/shi kong, which encodes filamin, disrupt actin filament organisation and so compromise membrane integrity (Li et al., 1999; Robinson et al., 1997). Before the dumping of nurse cell cytoplasm, F-actin cables are formed that radiate from the nurse cell nuclei to the cell cortex to ensure that nuclei do not block the ring canals. Such a blockage is observed in mutants for chickadee, which is required for F-actin polymerisation, and quail and singed, which both encode actin filament bundling proteins (Cant et al., 1994; Cooley et al., 1992; Mahajan-Miklos and Cooley, 1994). Whereas the earlier transfer of particles seems to be linked to basket-like actin structures at the ring canals (Riparbelli and Callaini, 1995), fast transfer of nurse cell cytoplasm is mediated by myosin II-dependent contraction of F-actin at the nurse cell cortex (Cooley et al., 1992; Edwards and Kiehart, 1996; Wheatley et al., 1995).

We undertook the present study to define domains of PavKLP that direct its subcellular localisation. Knowing that the protein mediates crucial interactions with the microtubule and actin cytoskeleton in dividing cells, we wished to know how its subcellular localisation was controlled as the oocyte forms in the transition between the mitotic divisions of the germarium and meiosis. To this end, we expressed mutant forms of PavKLP tagged with GFP in the female germ line. We show that several of these exhibit dominant mutant phenotypes during oogenesis that reflect a need to regulate the amounts and spatiotemporal distribution of the motor protein, and thereby its interactions with both the microtubule and the actin cytoskeleton during the development of the egg chamber.

\section{Materials and Methods}

Transformants carrying a ubiquitin-driven GFP-Pav-KLP construct

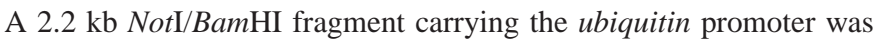

subcloned into pKS at the corresponding restriction sites. This construct (pKSUbNB) was first digested with NcoI, treated with Mung Bean Nuclease to remove the overhanging ATG within the NcoI site and re-ligated to generate pKSUbNB-NcoI. A $0.7 \mathrm{~kb} \mathrm{BamHI/SalI}$ fragment coding for the GFP variant mGFP6 (also known as MmGFP (Zernicka-Goetz et al., 1996)) was inserted into the corresponding sites to yield pKSUbGFP. Subsequently, a $3.1 \mathrm{~kb}$ blunt-ended NdeI/NotI cDNA fragment coding for Pavarotti was ligated into the blunt-ended SalI site of pKSUbGFP to give an intermediary plasmid, pKSUGP. A $6 \mathrm{~kb}$ NotI fragment was produced by cutting pKSUGP with ApaI, treating with Klenow enzyme to give blunt ends, redigesting with NotI and finally ligating a NotI linker to the bluntended ApaI site. This NotI fragment, carrying the poly ubiquitin promoter and the chimeric GFP and Pav-KLP genes, was subcloned into the corresponding site of the Drosophila transformation vector pCasper4 to produce cUGP.

This plasmid was introduced using standard procedures into $w^{1118}$ embryos. Surviving adults were mated individually with $w^{1118}$ virgin females or males, and progeny showing a mosaic $w^{+}$phenotype were identified as transformants. Two homozygous viable and fertile lines were eatablished in which the UGP construct had either inserted on the second $\left(w^{1118} ; p\left[w^{+} U b-G F P-P a v-K L P\right] 53\right)$ or third $\left(w^{1118} ;+; p\left[w^{+} U b-G F P-P a v-K L P\right] 31\right)$ chromosome. The insert on the second chromosome was able to rescue pav mutants.

\section{Transformants carrying UAS-GFP-Pav-KLP constructs Full-length and domain-specific wild-type Pav-KLP constructs}

The full-length $3.1 \mathrm{~kb}$ pav cDNA 40B15 was first subcloned into pET28a as an NdeI/NotI fragment to produce construct A1. A bluntended $0.7 \mathrm{~kb} B g l \mathrm{II} / \mathrm{SalI}$ DNA fragment encoding the GFP variant MmGFP was then inserted into the blunt-ended NdeI site of construct $A 1$ to produce construct $A 2$. This construct was subsequently digested with SmaI and NotI and the resulting $3.8 \mathrm{~kb}$ fragment, which included both the GFP and Pav-KLP genes, was then ligated into the corresponding sites of the cloning vector pSK to produce clone A3. The same SmaI/NotI fragment was also ligated into NotI and bluntended KpnI of the Drosophila transformation vector pUASp (Rorth, 1998) to produce the final construct pUASp-GFP-Pav-KLP (PGP).

The Alconstruct was digested with BsrGI and BssHII and then incubated with Klenow enzyme to remove overhanging nucleotides. A construct $B 1$ was produced by inserting the resulting blunt-ended $0.6 \mathrm{~kb}$ DNA fragment, which encodes the central stalk domain of Pav-KLP (Pavcoil), into the blunt-ended SalI site of the vector $\mathrm{p} \beta \mathrm{GFP} / \mathrm{RN} 3 \mathrm{P}+\mathrm{AT}$. This vector is based on the construct p $\beta$ GFP/RN3P (Zernicka-Goetz et al., 1996) and contains a 5'-XhoISalI-ApaI-HindIII-StuI-3' linker downstream of the MmGFP gene (A. Tavares, unpublished). Construct $B 1$ was digested with EcoRI and StuI to produce a $1.3 \mathrm{~kb}$ DNA fragment that includes the Pavcoil coding sequence downstream of the MmGFP gene. This fragment was subsequently inserted into EcoRI and blunt-ended XhoI of the other Drosophila transformation vector pUAST (Brand and Perrimon, 1993) to produce the construct pUAST-GFP-Pavcoil. This product was digested with $B a m \mathrm{HI}$ and $\mathrm{XbaI}$ and the resulting $1.3 \mathrm{~kb}$ GFPPavcoil fragment inserted into the corresponding sites of pUASp to produce the final construct pUASp-GFP-PavSTALK.

\section{Mutated full-length Pav-KLP constructs}

The QuickChange ${ }^{\mathrm{TM}}$ Site-Directed Mutagenesis Kit (Stratagene) was used to introduce point mutations into either the ATP-binding site of the motor domain or the $\mathrm{C}$-terminal nuclear localisation signals (NLSs) of Pavarotti (Fig. 2). Construct $A 3$ above was used as the initial template unless stated otherwise.

The G131E mutation was introduced into the ATP-binding site using the oligonucleotides GGCGTGACTGGAAGTGAGAAAA- 
CGTACACCATG and CATGGTGTACGTTTTCTCACTTCCAGTCACGCC. This mutated form of A3 was digested with SmaI and NotI and the resulting $3.8 \mathrm{~kb}$ DNA fragment inserted between the Not I and blunt ended KpnI sites of pUASp to produce pUASp-GFPPavDEAD.

A three amino-acid $\mathrm{K}_{770} \mathrm{NR}$ to AAA mutation was introduced into nuclear localisation sequence (NLS) 5 using the oligonucleotides CTATTATGCAGCCGTATCTGGCGGCGGCGAAATCCGTAACG AAACTAAC and GTTAGTTTCGTTACGGATTTCGCCGCCGCCAGATACGGCTGCATAATAG. The resulting mutant SmaI and NotI fragment was purified and inserted into the NotI and blunt-ended KpnI sites of pUASp to produce pUASp-GFP-PavNLS5*

Successive rounds of mutagenesis were carried out on the previous mutant fragment to introduce first the $\mathrm{K}_{770} \mathrm{NR}$ to AAA mutation and then the $\mathrm{R}_{843} \mathrm{KR}$ to AAA mutation. This utilised the following respective pairs of oligonucleotides: CTATTATGCAGCCGTATCTGGCGGCGGCGAAATCCGTAACGAAACTAAC and GTTAGTTTCGTTACGGATTTCGCCGCCGCCAGATACGGCTGCATAATA $\mathrm{G}$; and CCGGTTCACTCGCCCACGGCGGCGGCGCCCAGCAATGGCAACATTTCG and CGAAATGTTGCCATTGCTGGGCGCCGCCGCCGTGGGCGAGTGAACCGG. The final product was inserted into NotI and blunt-ended KpnI of pUASp to produce the final construct pUASp-GFP-PavNLS(4-7)*.

The UASp constructs were transformed into $w^{1118}$ flies using standard approaches and several homozygous viable and fertile transformants for each of the construct were obtained.

\section{Immunocytochemistry}

For Hoechst or rhodamine-phalloidin staining, ovaries were dissected in PBS, fixed for 15 minutes in 5\% Paraformaldehyde (PFA) in PBS, rinsed extensively with PBS and then incubated in either $0.5 \mu \mathrm{g} / \mathrm{ml}$ Hoechst or $5 \mathrm{u} / \mathrm{ml}$ rhodamine-phalloidin (Molecular Probes) in PBS for 20 minutes. After several washes with PBS, ovaries were mounted in Vectashield mounting medium H-1000 (Vector Laboratories) and visualized by confocal microscopy (BioRad MRC 1024).

For immunostaining, ovaries were dissected in TriPBS $(0.2 \%$ Triton-X100 in PBS), washed $3 \times$ with PBS and then incubated for 45 seconds in one volume of freshly prepared PBS, $1.3 \%$ PFA and 1.25 volumes Heptane under vigorous shaking. Fixation was done in PBS, $3.3 \%$ PFA, 5\% DMSO for 20 minutes. The ovaries were washed twice with methanol prior to incubating them in methanol containing $2 \%$ hydrogen peroxide for 30 minutes. After a short wash in TriPBS, the ovaries were first blocked in TriPBS, 5\% FCS for 1 to 3 hours and then incubated overnight at $4^{\circ} \mathrm{C}$ in primary antibody diluted in TriPBS (anti- $\alpha$-tubulin YL1/2 (Harlan Sera lab) at 1/20; anti-Pavarotti Rb3301 (Adams et al., 1998) at 1/250, anti-Hu-li tai shao 6554A (Robinson et al., 1994) at 1/1, anti-Staufen (St Johnston et al., 1991) at 1/3000, anti-Orbit (Inoue et al., 2000) at 1/200, anti-Mini spindles (Cullen et al., 1999) at 1/500 and anti-Myosin II 656 (Kiehart and Feghali, 1986) at 1/1000). Ovaries were washed twice in TriPBS and then incubated in secondary antibody diluted in TriPBS for 2 to 3 hours. After two wash steps with TriPBS and an additional one with PBS, ovaries were incubated in PBS containing $1 \mu \mathrm{M}$ TOTO-3 (Molecular Probes) for 45 minutes to stain DNA. The ovaries were finally mounted in Vectashield mounting medium H-1000 (Vector Laboratories) overnight at $4^{\circ} \mathrm{C}$ before visualizing them by confocal microscopy (BioRad MRC 1024).

\section{Germ-line clone analysis of the pav-klp}

In order to study germ line pav clones, we recombined pav onto an FRT chromosome and then generated females of the genotype $y w$ FLP ${ }^{22}$; pav-klp, FRT/TM3 which we crossed to $w / Y$; ovo ${ }^{D 1}, F R T / T M 3$ males. Eggs were collected daily over a period of 16 days and the progeny subjected to a 2 hour heat shock at $37^{\circ} \mathrm{C}$ on two consecutive days during their late L2 to L3 larval stages. The adult yw FLP22; pav- $k l p, F R T / o v o^{D 1}, F R T$ female progeny were then checked for fertility over a period of 5 days. The ovo ${ }^{\mathrm{D} 1}$ mutation arrests oogenesis at stage 3-4. Thus ovaries of $y w F L P^{22}$; pav-klp, FRT/ ovo ${ }^{D 1}$, FRT females should not form mature egg chambers. Eggs were also not produced after heat shock, indicating that homozygous pav-klp, FRT clones do not permit egg formation. In a parallel experiment with vihar, a gene encoding an E2 ubiquitin-conjugating enzyme essential for the development of the syncytial embryo, we identified approximately $10 \%$ of females as egg laying. In each case we examined about 1000 females for fertility.

\section{Results}

Pav-KLP localises to nurse cell and follicle cell ring canals and accumulates in the oocyte nucleus

We have previously shown that Pav-KLP becomes associated with the ring canals, which form at the sites of incomplete cytokinesis in the cysts of spermatocytes (Carmena et al., 1998). To determine whether it also accumulates in comparable structures during oogenesis, we localised the protein both by immunostaining ovaries of wild-type flies and examining the fluorescence of ovaries dissected from flies expressing a GFP-
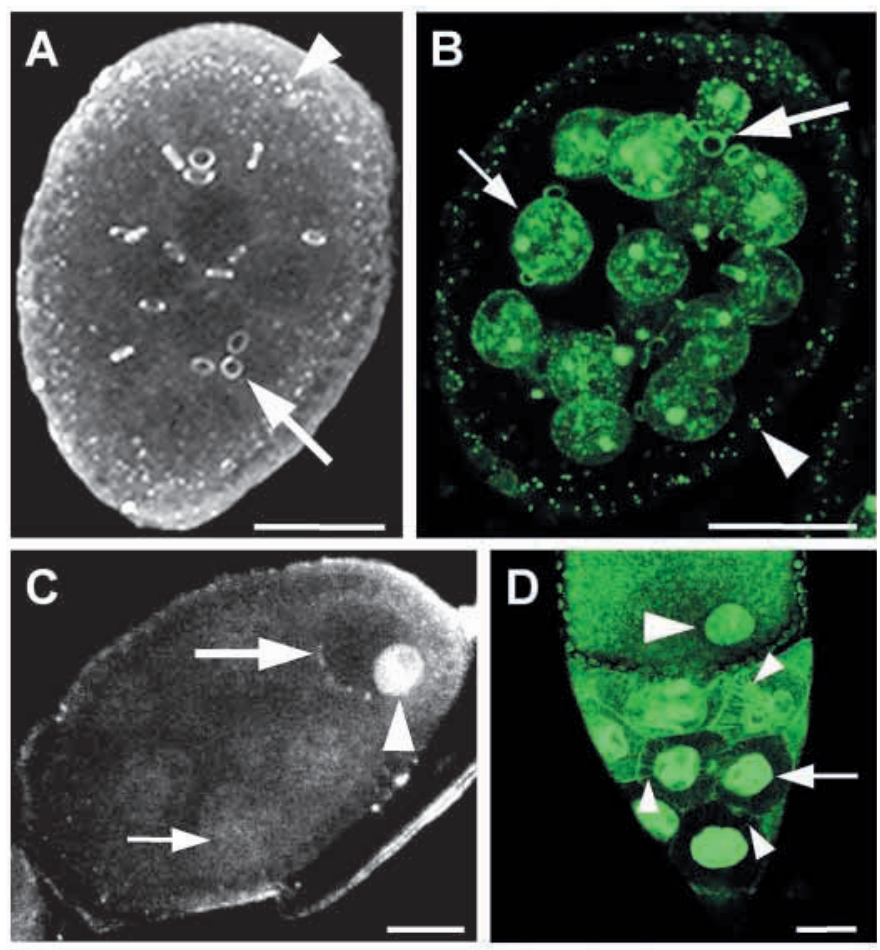

Fig. 1. Comparison of the immunolocalisation of Pav-KLP $(A, C)$ with the localisation of GFP-tagged Pav-KLP expressed from the poly ubiquitin promoter (B,D). (A,B) In early cysts both Pav-KLP and GFP-Pav-KLP accumulate in the ring canals that connect either the germ cells (arrow in A, large arrow in B) or the follicular cells (arrowheads in A and B) with each other. GFP-Pav-KLP is also present in germ cell nuclei (small arrow in B). (C,D) At a later stage, both Pav-KLP and GFP-Pav-KLP concentrate in the oocyte nucleus (arrowhead in $\mathrm{C}$ and large arrowhead in D) and persist in the ring canals (large arrow in C, not visible in D). Nurse cell nuclei accumulate much higher concentrations of GFP-Pav-KLP (arrow in D) than Pav-KLP (small arrow in C). GFP-Pav-KLP is also found along actin filament bundles from stage 10B through 11 (small arrowheads in D). Bars, $20 \mu \mathrm{m}$ (A, B, C) and $50 \mu \mathrm{m}$ (D). 
tagged transgene. Immunostaining revealed Pav-KLP in the 15 ring canals that connect the 16 germ line cells throughout oogenesis (arrow, Fig. 1A; large arrow, Fig. 1C) and in a punctate pattern at the surface of early egg chambers (arrowhead, Fig. 1A). The latter are cytoplasmic bridges associated with the smaller ring canals on follicle cells (Giorgi, 1978). In stage 7 egg chambers, Pav-KLP was prominent within the oocyte nucleus (arrowhead, Fig. 1C) and was found at lower levels in nurse cell nuclei (small arrow, Fig. 1C). We observed a similar pattern of localisation in ovaries of flies expressing a gfp-pavarotti transgene driven from the polyubiquitin promoter that is fully able to rescue pav mutants (see Materials and Methods). In this line we first detected GFPPav-KLP in cystoblasts in region 1 of the germarium at the anterior tip of the ovariole and subsequently in the cleavage rings of mitotically dividing cysts (not shown). Stage 5 egg chambers showed GFP-Pav-KLP in the 15 ring canals in the germ line cyst (large arrow, Fig. 1B) and in the ring canals of the follicular cells (arrowhead, Fig. 1B). In addition the protein appeared to be present in discrete spots at higher levels within nurse cell nuclei (small arrow, Fig. 1B) than revealed by immunostaining for the endogenous protein in wild-type ovaries. By the time the egg chambers reached stage 10B, the speckled localisation pattern of GFP-Pav-KLP within nurse cell nuclei had changed to a dense network (arrow, Fig. 1D), and the concentration of the motor protein within the oocyte nucleus had increased (large arrowhead, Fig. 1D). GFP-PavKLP also associated with microfilaments extending from the cortex of nurse cells to their nuclei (small arrowheads, Fig. 1D). Western blotting suggested that nuclear accumulation of Pav-GFP is likely to be a consequence of its higher level of expression of the transgene than the endogenous pav gene in ovaries.

\section{Overexpression of wild-type GFP-Pav-KLP disrupts nurse cell dumping}

All extant pavarotti mutant alleles arrest cell division in cycle 16 of embryogenesis as a result of the failure of cytokinesis (Adams et al., 1998). Thus in order to observe the effect of such mutations upon cell division at later stages of development, it is necessary to generate homozygous mutant clones of cells. When we used the $o v o^{D 1}$ system (Chou et al., 1993) to generate clones of homozygous pav cells in the germ line we were unable to detect the production of eggs or indeed any development beyond oogenesis stage 3-4 when ovo ${ }^{D 1}$ itself causes arrest. Thus pav appears to be essential for the early stages of oogenesis, most probably for cell division in the germarium. We therefore turned to address the effects of overexpressing wild-type and mutant forms of Pav-KLP in the female germ line. The expression of GFP-Pav-KLP in ovaries from the polyubiquitin promoter emphasised two aspects of localisation of the protein that are also seen in proliferating cells, namely its localisation to interphase nuclei and to the remnants of the central telophase spindle known as the mid-bodies (Adams et al., 1998). We wished to determine which domains within the protein were responsible for these features of localisation and to this end chose to construct and express GFP-tagged mutant and wild-type forms of the gene introduced as transgenes into flies. Conscious that the expression of such mutant proteins may lead to lethality, we chose to use a variant on the GAL4/UAS conditional system

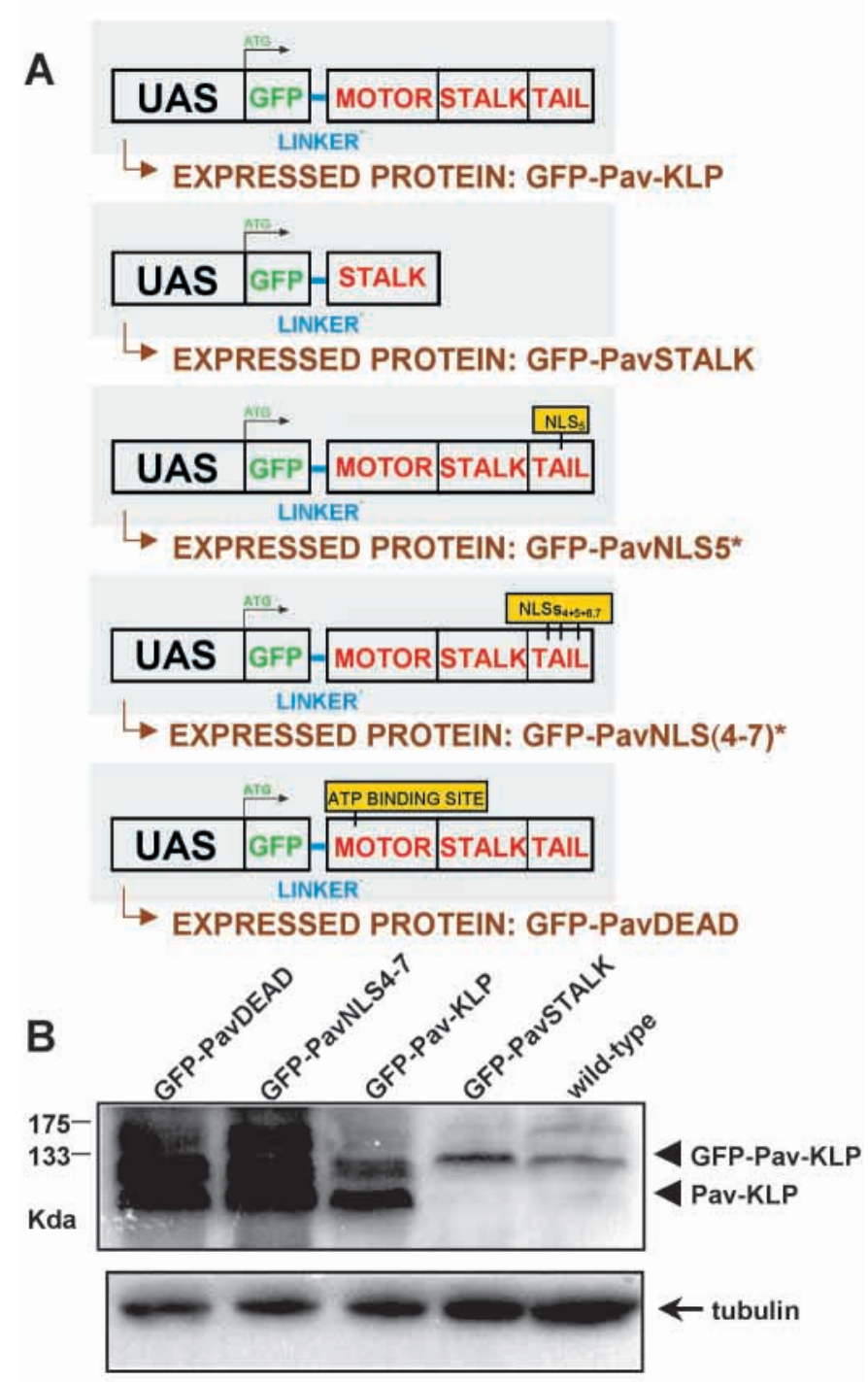

Fig. 2. Wild-type and mutant forms of Pav-KLP introduced into the Drosophila germ line under UAS control. (A) Different GFP-tagged Pavarotti variants were subcloned into the Drosophila transformation vector pUASp for expression in the female germ line. Regions subjected to site-directed mutagenesis are indicated in yellow boxes. Linking amino acids between the GFP tag and domains of Pavarotti are represented as a blue bar. The first ATG to be translated is given in green. The proteins that derive from these constructs and are used in the text and figures are given below each drawing. (B) Western blots to indicate levels of expression of the indicated constructs in ovaries when driven by GAL4-VP16 under control of the maternal $\alpha 4$-tubulin promoter as described in the text.

(Brand and Perrimon, 1993) that enables germ line expression during oogenesis (Rorth, 1998). Flies carrying the appropriate mutant transgenes in the pUASp vector were then crossed with flies carrying GAL4-VP16 (Sadowski et al., 1988) under control of the maternal $\alpha 4$-tubulin promoter (a kind gift of D. St Johnston). We used this system to express full-length wild-type Pav-KLP and several mutant forms each fused to GFP to facilitate localisation of the encoded protein. The construction of mutants was influenced by a preliminary report that the stalk domain of MKLP-1 appears to target the motor protein to the midzone of mammalian mitotic spindles (Matuliene and 

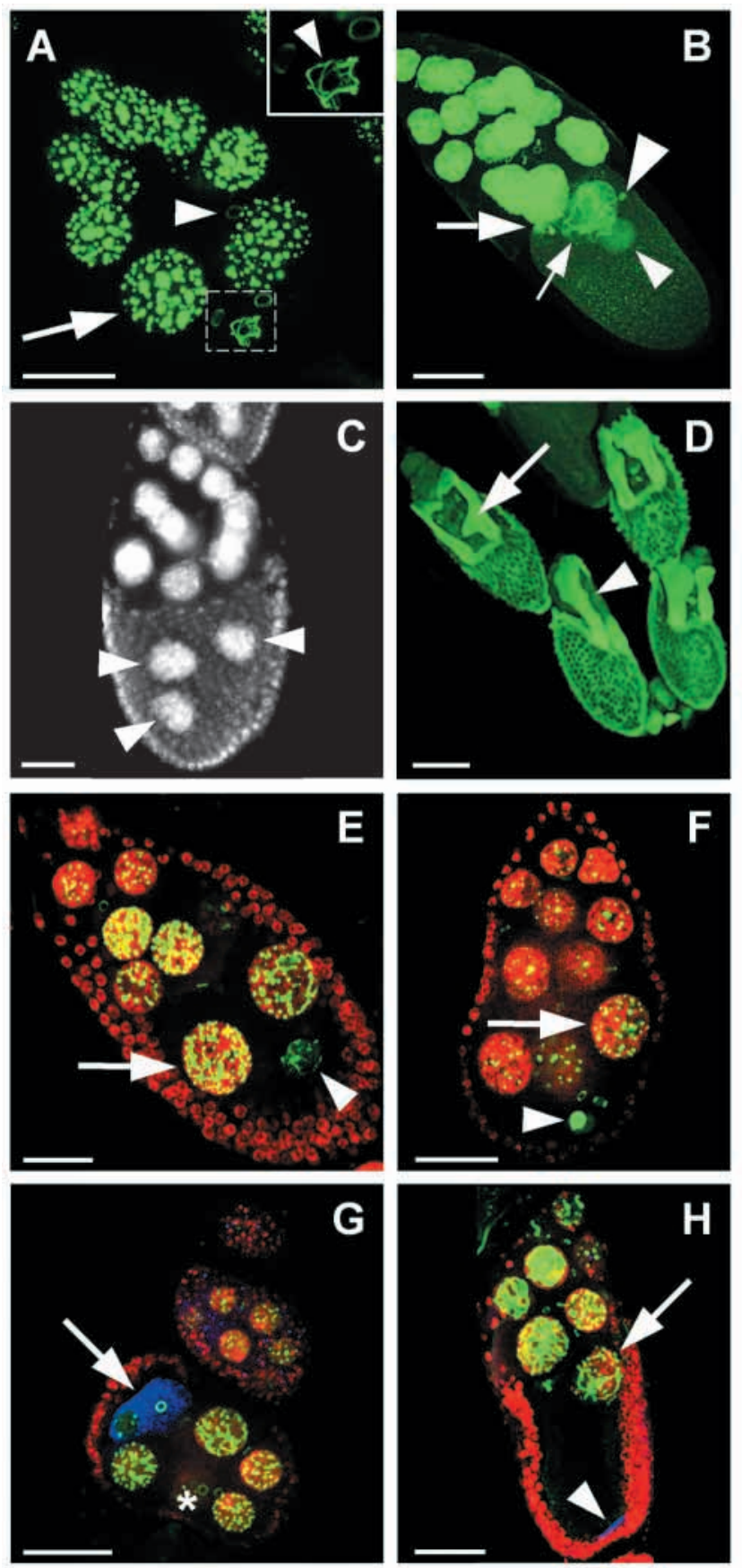

Kuriyama, 1998) and that sequences in the C-terminal domain of MKLP-1 specify its nuclear localisation (Deavours and Walker, 1999). We therefore constructed a gene encoding only the central stalk region of the molecule fused to GFP (GFPPavSTALK) and several variants of the full-length Pav-KLP molecule containing mutations in putative nuclear localisation sequences (NLSs) in its C-terminus (GFP-PavKLPNLS5* and GFP-PavKLPNLS(4-7)*). It had also been shown that mutations in a conserved ATP-binding domain of Kar3 protein, a yeast kinesin-related motor, caused the mutant protein to bind and stabilise cytoplasmic microtubules (Meluh and Rose, 1990). We therefore constructed an equivalent mutation in the ATP-binding site of Pav-KLP that we expected would direct the synthesis of an inactive protein (GFP-PavDEAD) (Fig. 2). We have examined
Fig. 3. Defects in oogenesis resulting from overexpression of wildtype Pav-KLP. Live (A,B,D), Hoechst-stained (C) or immunostained (E-H) egg chambers expressing GFP-Pav-KLP (green) are shown. DNA is shown in red (E-H) and Staufen in blue $(\mathrm{G}-\mathrm{H})$. (A) Stage 5 oocyte with GFP-Pav-KLP in speckles within nurse cell nuclei (arrow); GFP-Pav-KLP is also present in ring canals (arrowhead). The oocyte nucleus appears to contain a filamentous network (dashed box and arrowhead in inset). (B) An egg chamber in which the oocyte nucleus (small arrowhead) is being pushed towards the posterior pole by an invading nurse cell (small arrow), which has been able to pass the nurse cell/oocyte border (large arrow). The large arrowhead points at one of the four ring canals that are normally located at the nurse cell/oocyte border. (C) Egg chamber in which the oocyte at the posterior half has been invaded by three nurse cell nuclei (arrowheads). (D) Dumpless egg chambers with nurse cells that have retained most of their cytoplasm (arrowhead). Although dorsal appendage follicle cells have migrated more or less normally, the appendages (revealed by auto-fluorescence) are broad and irregular in shape (arrow). (E,F) Egg chambers isolated from females fed for 24 hours on unsupplemented yeast paste (E) or yeast paste containing 1 $\mathrm{mg} / \mathrm{ml}$ colchicine $(\mathrm{F})$. Egg chambers isolated from untreated females contain nurse cells that have a clearly visible nuclear GFP-Pav-KLP network (arrow in E). A similar network is also associated with the oocyte nucleus (arrowhead in E). In colchicine-treated egg chambers GFP-Pav-KLP is still present in nurse cell nuclei, but it does not form filaments any more (arrow in F). The network associated with the oocyte nucleus has also collapsed (arrowhead in F).

(G) Overexpression of GFP-Pav-KLP can lead to egg chambers, in which the oocyte (arrow) fails to localise to the posterior pole (asterisk). (H) An egg chamber in which one nurse cell (arrow) has passed the nurse cell/oocyte border without affecting Staufen localisation at the posterior pole of the oocyte (arrowhead). Bars, 20 $\mu \mathrm{m}$ (A), $50 \mu \mathrm{m}$ (C,E-H), $100 \mu \mathrm{m}$ (B) and $200 \mu \mathrm{m}$ (D).

the consequences of the expression of each of these proteins in females carrying one copy of the UASp construct and one copy of the $\alpha 4$-tubulin-driven GAL4-VP16 transgene.

Whereas expression of the wild-type Pav-KLP tagged with GFP from the polyubiquitin promoter permitted the development of fertile females, we found expression of the equivalent protein from the GAL4-VP16/UASp system resulted in female sterility. This was associated with an increase in expression level by 5-10 fold over wild-type as indicated by western blots on whole ovaries (Fig. 2B). We observed that a common defect in the ovaries of these sterile flies was that nurse cells were not contained within the anterior half of the egg chamber, instead whole nurse cells or nurse cell nuclei protruded into the oocyte compartment (Fig. 3B,C). Moreover such mislocalised nurse cells did not regress as normally occurs when nurse cells dump their contents, so leading to the formation of eggs with several large nuclei. In up to $40 \%$ of egg chambers there was no fast cytoplasmic streaming from the nurse cells into the oocyte, the nurse cells failed to regress, and eggs never reached full size (Fig. 3D). Most stage 2 to $10 \mathrm{~A}$ egg chambers overexpressing the protein contained a speckled distribution of GFP-Pav-KLP within their nurse cell nuclei (arrow, Fig. 3A) and ring canals (arrowhead, Fig. 3A). This frequently developed into a filamentous network in the nuclei of oocytes (inset, Fig. 3A and arrowhead, Fig. 3E) and nurse cells (small arrow, Fig. 3B and arrow, Fig. 3E). These filamentous structures were not stained with antibodies against tubulin or myosin or by the F-actin-binding toxin phalloidin. Nevertheless, knowing the Pav-KLP can interact with 
HU-LI TAI SHAO

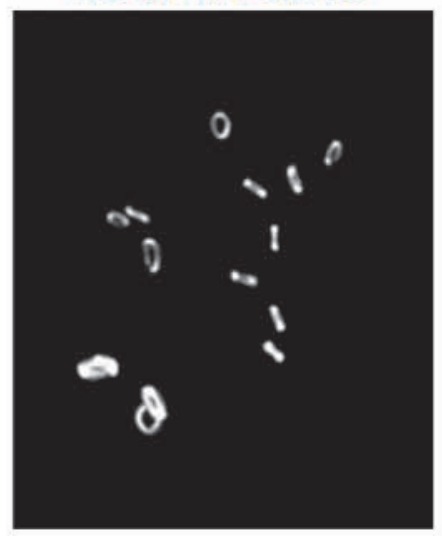

GFP-PavSTALK

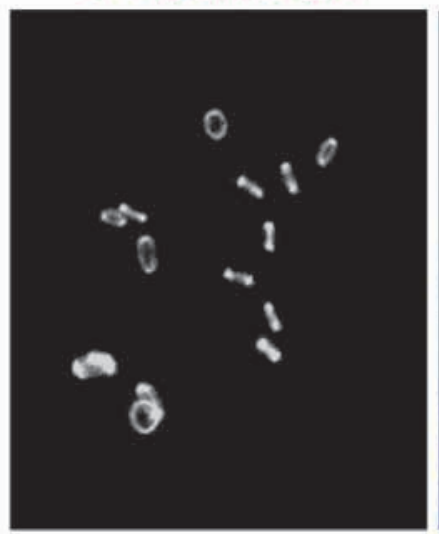

MERGE

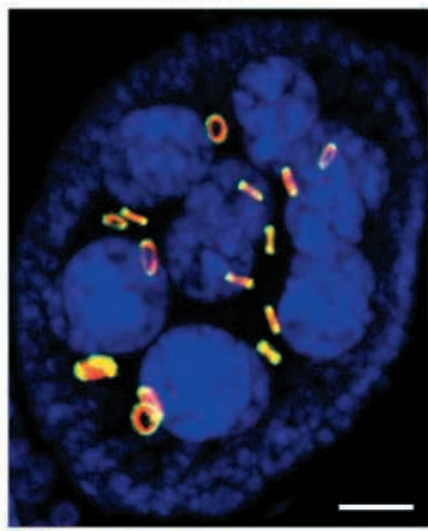

Fig. 4. Localisation of the GFPtagged stalk domain of Pav-KLP to ring canals. An egg chamber expressing GFP-PavSTALK (green) and stained for $\mathrm{Hu}$-li tai shao (red) and DNA (blue). GFP-PavSTALK appears to be localised at the outer side or ring canals, whereas $\mathrm{Hu}-\mathrm{li}$ tai shao is more concentrated at the inner side. Regions where the two proteins overlap appear yellow. Bar, $10 \mu \mathrm{m}$. microtubules we wished to investigate whether the network was sensitive to colchicine and so isolated ovarioles from females fed with $1 \mathrm{mg} / \mathrm{ml}$ of this drug for 24 hours. The egg chambers from such ovarioles contained only a punctate GFPPav-KLP fluorescence in their nurse cell nuclei (arrow, Fig. 3 F) and uniform fluorescence in the oocyte nucleus (arrowhead, Fig. 3F). This colchicine sensitivity implies that the network is microtubule based. It suggests that tubulin may be drawn into the nuclei by the abundance of the Pav-KLP protein in which case the failure of anti-tubulin antibodies to recognise the structure might be due to the steric hindrance imposed by the high levels of the motor protein. However, other explanations cannot be discounted, and it is possible that destabilisation of cytoplasmic microtubules may result in failure of some other protein essential for the stability of the nuclear filaments to associate with the nucleus.

We also found that the oocyte (identified by Staufen localisation) was often misplaced within earlier stage egg chambers. In wild-type egg chambers, the oocyte moves around the cortex in region 2 of the germarium to reach its final destination at the posterior. Egg chambers overexpressing GFPPav-KLP sometimes had an oocyte that was not positioned at the posterior (Fig. 3G). Nevertheless, stage 10 egg chambers containing nurse cells within the oocyte compartment (arrow, Fig. $3 \mathrm{H}$ ) were still able to localise Staufen protein properly at the posterior pole (arrowhead, Fig. 3H), indicating that some intercellular transport functions were still intact.

\section{The central stalk of Pav-KLP is responsible for its} localisation to the outer rim of ring canals

In contrast to the female sterility seen when full-length PavKLP is overexpressed using the GAL4-VP16/UASp system, females expressing only the stalk domain fused to GFP in this way were fully viable and fertile. Strikingly, the fusion protein was specifically seen in the 15 ring canals connecting the nurse cells and the oocyte at all stages of oogenesis (Fig. 4). When expression was driven by daughterless-GAL4, we also found staining in the cytoplasmic bridges between follicular cells (not shown). This suggests that the coiled coil domain alone is sufficient for ring canal localisation of Pav-KLP. We looked to see when and where GFP-PavSTALK localised to the ring canals with respect to either the $\mathrm{Hu}$-li tai shao isoform ADD-60 and actin (Theurkauf et al., 1993; Tilney et al., 1996;
Warn et al., 1985; Yue and Spradling, 1992; Zaccai and Lipshitz, 1996a; Zaccai and Lipshitz, 1996b). Both of these proteins are recruited to ring canals at the same time in region $2 \mathrm{a}$ of the germarium and are enriched toward the inner surface of the ring canal (Robinson et al., 1994). We found that in stage 3-5 egg chambers GFP-PavSTALK fluorescence always surrounded $\mathrm{Hu}$-li tai shao (Fig. 4) or actin (not shown) staining, suggesting that GFP-Pav-KLP is a component of the outer rim.

\section{Mutant Pav-KLPs incapable of nuclear localisation or putatively immotile stabilise cytoplasmic microtubules}

In dividing cells, members of the MKLP-1 family of kinesinlike proteins associate with microtubules only during mitosis; during interphase they are found in the nucleus except $\mathrm{KRP}_{110}$, which shows a perinuclear distribution (Chui et al., 2000). In the founder member of the family, mammalian MKLP-1, this appears to be mediated by a classic nuclear localisation sequences (NLSs) in the C-terminal domain of the molecule (Deavours and Walker, 1999). The equivalent region of PavKLP appeared to contain four out of seven such NLS motifs recognised by the PSORT II program (http://psort.nibb.ac.jp/), two of which (NLS 6 and 7) were overlapping. To determine whether these sequences were responsible for the nuclear localisation of Pav-KLP we made mutations in either NLS 5 or NLS elements 4-7 (Materials and Methods) and placed the mutant genes tagged with GFP downstream of the UAS regulatory element (GFP-PavNLS5* and GFP-PavNLS(4-7)* respectively) (Fig. 2). When expressed using the GAL4VP16/UASp system we found that GFP-PavNLS5* showed the same distribution as the GFP-tagged wild-type protein and was most abundant in the nurse cell and oocyte nuclei (arrow, Fig. 5A) and in the ring canals (arrowhead, Fig. 5A). This indicates that NLS 5 is not required for nuclear localisation. Females showing overexpression of GFP-PavNLS(4-7)* in their ovaries were completely sterile and showed no GFP fluorescence in any nuclei (Fig. 5B,C). Instead they showed very strong filamentous GFP fluorescence in the cytoplasm (arrowhead, Fig. 5B) and at the cortex of nurse cells as well as in ring canals. These filaments correspond to microtubules that appear to be more stable than usual microtubules as feeding flies yeast paste containing $50 \mu \mathrm{g} / \mathrm{ml}$ colchicine for 48 hours resulted only in their partial depolymerisation. A complete disruption of the 
network was achieved however, following feeding with up to $1 \mathrm{mg} / \mathrm{ml}$ of colchicine for 24 hours (data not shown).

We also observed similar defects following the overexpression of a variant of Pav-KLP with a mutation in the ATP-binding site of the motor domain (GFP-PavDEAD) (Fig. 2). Females overexpressing this molecule were completely sterile and did not lay any eggs. GFP fluorescence was associated with a filamentous cytoplasmic network and ring canals but was not seen within nuclei (arrow, Fig. 5D). This cytoplasmic network appears to become denser as egg chambers become older leading to an accumulation of nurse cell nuclei in the middle of the egg chamber (arrowhead, Fig. 5D). In lines expressing GFP-PavNLS(4-7)* or GFPPavDEAD, the early stages of nurse cell development and the specification of the oocyte did not appear to be affected. Immunostaining to reveal the Staufen protein indicated that each egg chamber developed a single oocyte, but nevertheless this cell became mispositioned (arrowhead, Fig. 7B) as we had observed following the overexpression of GFP-Pav-KLP (Fig. $3 \mathrm{G})$. However, whereas at later stages in wild-type oogenesis the nurse cells (arrowhead, Fig. 5E) regressed concomitantly with oocyte growth (arrow, Fig. 5E), they failed to do so in egg chambers expressing GFP-PavDEAD, which rarely develop beyond stage 10 (arrowheads, Fig. 5F).

To understand further the properties of the cytoplasmic network seen following expression of either GFP-PavDEAD or GFP-PavNLS(4-7)*, we asked whether the filaments contained tubulin and whether they were associated with two microtubuleassociated proteins (MAPs) encoded by the orbit (Inoue et al., 2000) and mini spindles (msps) (Cullen et al., 1999) genes. We found extensive colocalisation of GFP-PavDEAD with both tubulin and the Orbit protein along the cytoplasmic filaments (Fig. 6A,D; Fig. B,E, respectively). In contrast, Msps protein did not bind to the filaments in spite of a high cytoplasmic concentration of the protein (arrow, Fig. 6C,F). Similar results were obtained in ovaries overexpressing GFP-PavNLS(4-7)* (data not shown). This indicates that the filaments contain microtubules to which the two MAPs show differing affinities. This may reflect an ability of Orbit to bind to both interphase and M-phase microtubules in contrast to Msps having a preference for M-phase ones. Alternatively, GFP-PavDEAD may compete with Msps for microtubule binding. We found, in contrast to wild-type, that egg chamber microtubules in flies overexpressing GFP-PavDEAD could only be partially disrupted by feeding yeast paste containing $50 \mu \mathrm{g} / \mathrm{ml}$ colchicine. Following such treatment, microtubules were found predominantly at the nurse cell cortex (arrow, Fig. 6H), suggesting the possibility of stabilising interactions with molecules at the cortex such as actin (see also below). The oocyte remained void of microtubules following this treatment (arrowheads, Fig. 6G,H). A concentration of $1 \mathrm{mg} / \mathrm{ml}$ of the drug was required in the food to substantially destabilise microtubules, and even at this concentration there are residual microtubules that surround the nurse cell nuclei (arrow, Fig. 6I).

Overexpression of putatively immotile Pav-KLP leads to loss of cortical actin, membrane breakdown and formation of cytoskeletal aggregates

We were frequently able to observe egg chambers containing 32 cells in the ovaries of flies overexpressing GFP-PavDEAD or GFP-PavNLS(4-7)*. These could be seen at early stages (germarial region 3, Fig. 7A) when we also observed defects in oocyte positioning (Fig. 7B), and also at later stages of oogenesis (Fig. 7D-F). The origins of such egg chambers could be explained either by fusion of two chambers or by an
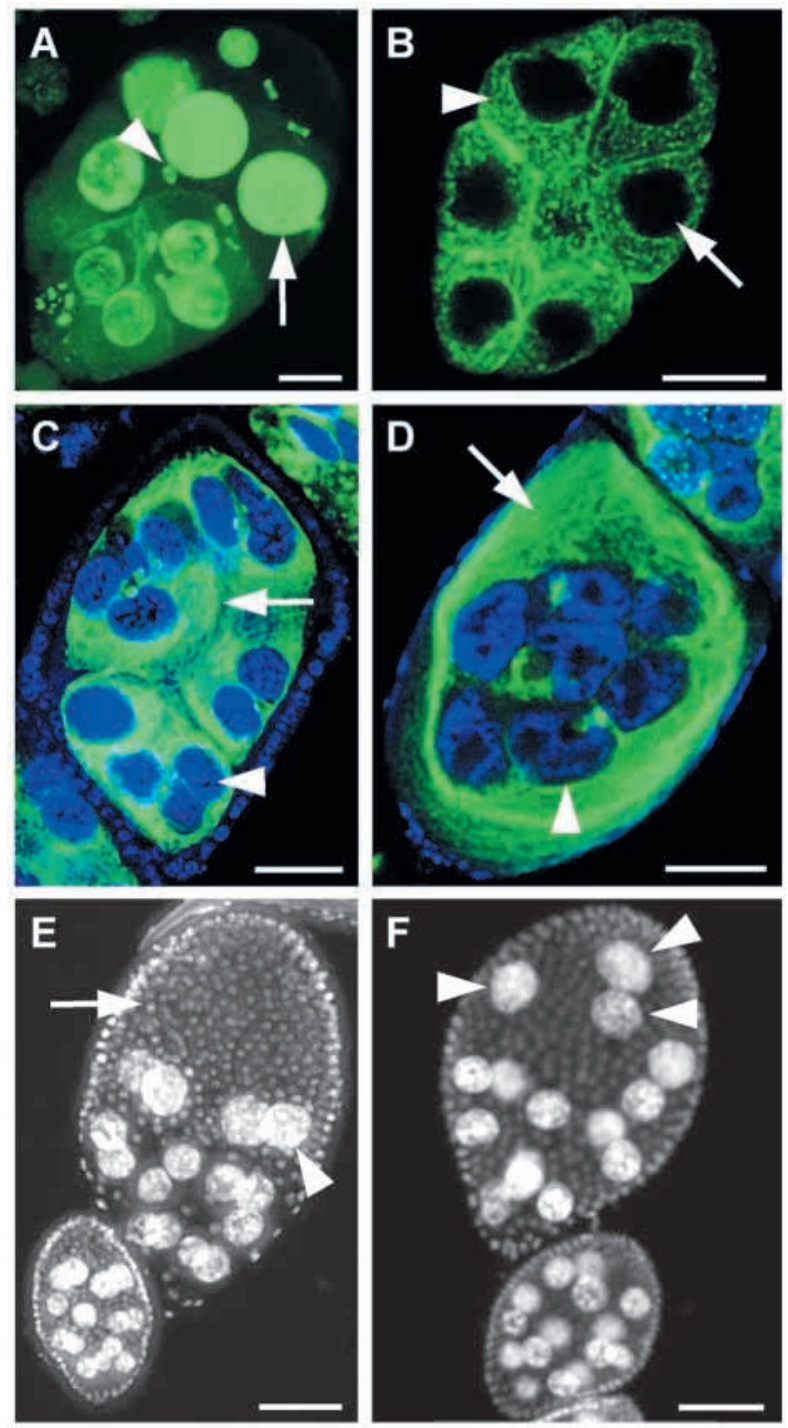

Fig. 5. Stabilisation of cytoplasmic microtubules and disorganisation of nurse cell positioning following overexpression of GFP-tagged PavDEAD or PavNLS(4-7)*. Live (A,B) or fixed (C-F) egg chambers expressing either GFP-PavNLS5* (A), GFP-PavNLS(4-7)* $(\mathrm{B}, \mathrm{C})$ or GFP-PavDEAD (D,F) (green). DNA was stained with TOTO-3 (blue, C,D) or Hoechst (E-F). (A) High concentrations of GFP-PavNLS5* are present in the germ cell nuclei (arrow) and in ring canals (arrowhead). (B) GFP-PavNLS(4-7)* is excluded from the nurse cell nuclei (arrow) and accumulates in the nurse cell cytoplasm (arrowhead). (C,D) Egg chambers in which nurse cell nuclei (arrowheads) are clustered and surrounded by a dense filamentous network (arrows) associated with GFP-PavNLS(4-7)* (C) or GFP-PavDEAD (D). (E) A wild-type egg chamber in which the follicular cells have started to migrate to the posterior pole (arrow). The 15 polyploid nurse cell nuclei are all localised outside of the oocyte at the anterior half of the egg chamber (arrowhead). (F) An egg chamber containing mispositioned nurse cells (arrowheads) following GFP-PavDEAD expression. Bars, $20 \mu \mathrm{m}$ $(\mathrm{A}, \mathrm{B}, \mathrm{D}, \mathrm{F})$ and $50 \mu \mathrm{m}(\mathrm{C}, \mathrm{E})$. 
extra round of mitosis in the germarium, as occurs in encore mutants (Hawkins et al., 1996). We were able to eliminate the latter possibility by counting the number of ring canals in each egg chamber. Egg chambers arising because of an additional fifth round of mitosis would have two cells with 5 ring canals only one of which would become an oocyte. Instead all of the 32-cell cysts in the dominant Pav-KLP mutants had two independent oocytes each with 4 ring canals indicating that fusion of egg chambers had occurred (Fig. 7E). Staining of ovarioles with fluorescent phalloidin revealed a number of localised defects in the actin cytoskeleton in these mutant flies. In the wild-type egg chamber, F-actin filaments are conspicuous in the nurse cell boundaries (arrowhead, Fig. 7C) and especially at the oocyte cortex (OC). Fused egg chambers showed varying degrees of loss of the actin cytoskeleton. When fusion had taken place early in oogenesis, all nurse cells within the cyst were similar in size (Fig. 7E). However we also observed cysts containing fused egg chambers of different ages where, for example, the older one (cyst 1 in Fig. 7F) had higher levels of the GFPtagged protein (in this case PavNLS(4-7)*) associated with the microtubular network. The older egg chamber in this fused cyst is particularly interesting as it exemplifies the collapse of ring canals (RC) and breakdown of the actin cytoskeleton between nurse cells (arrowhead) that we observed in both fused and non-fused egg chambers at this stage. It also reveals the colocalisation of microtubules with cortical actin. Thus the accumulation of GFP-Pav in the cytoplasm on stabilised microtubules appears to disrupt the organisation of cortical actin and thereby compromises membrane integrity. In its extreme form all of the internal membranes of the egg chamber collapse and both actin and tubulin accumulate in large aggregates (Fig. 7G).

Since actin filaments are also an important component of ovarian ring canals (Theurkauf et al., 1993; Tilney et al., 1996; Warn et al., 1985), and these are linked to the plasma membrane, it was of interest to know whether the accumulation of the dominant mutant forms of Pav-KLP had any effect on their structure. Towards this end we examined the localisation of the adducin homologue $\mathrm{Hu}$-li tai shao. In contrast to wild-type egg chambers, where the ADD-60 isoform of Hu-li tai shao is only localised in ring canals (arrowhead, Fig. 7H), we found that in early egg chambers overexpressing GFP-PavDEAD it was also present in the cortical cytoplasm of nurse cells (arrow, Fig. 7I), showing a similar distribution to cortical actin. We also found that the actin-tubulin aggregates that accumulate in the egg chambers once cell membranes have broken down also contain the Hu-li tai shao protein (data not shown). This appears to occur in concert with the progressive breakdown of the ring canal structures. Thus cytoplasmic accumulation of Pav-KLP either as a consequence of inactivating the motor or mutating nuclear localisation signals appears not only to result in the accumulation of stable arrays of cytoplasmic microtubules but also leads to the progressive disruption of the actin cytoskeleton.

\section{Discussion}

Pavarotti, a member of the MKLP-1 subfamily of kinesin-like proteins, is essential for cytokinesis. It has been proposed that it fulfils this role by correctly establishing the structure of the
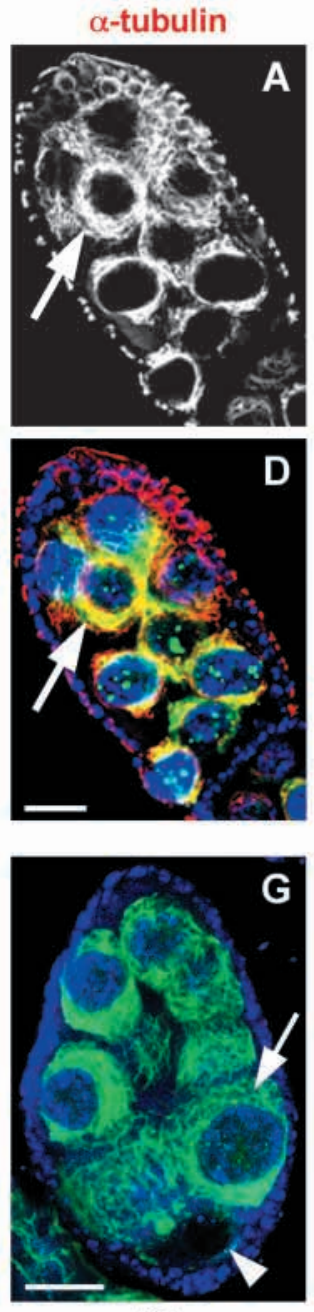

no

colchicine
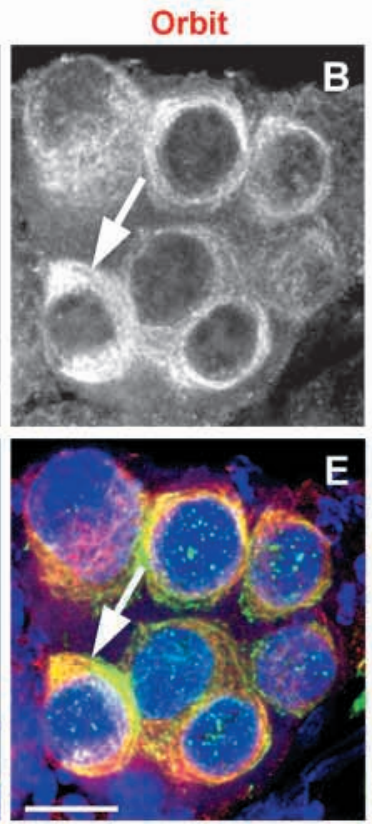

E
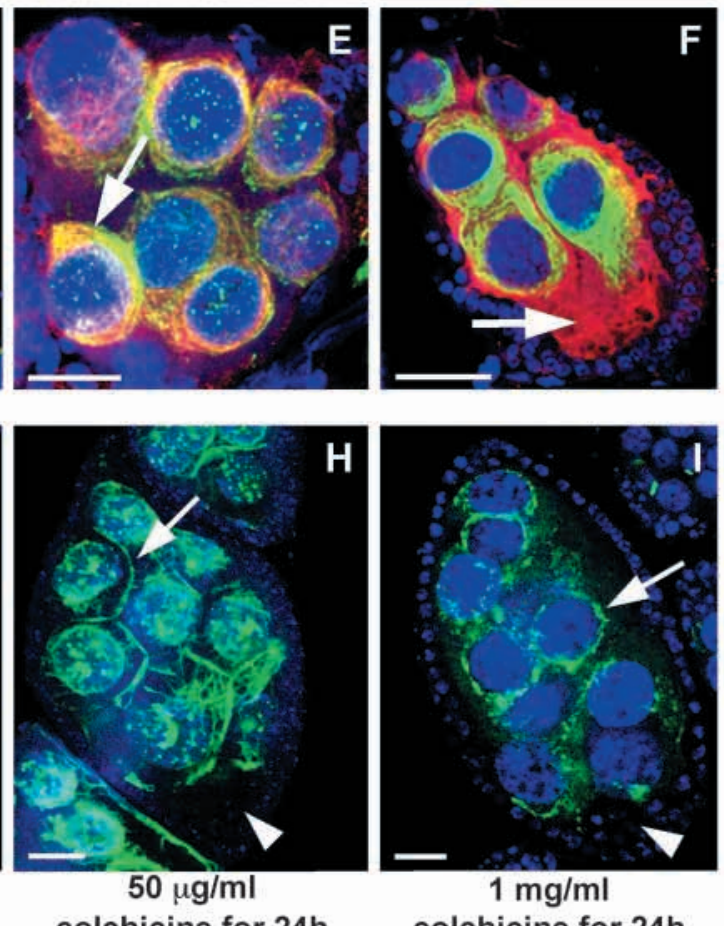

colchicine for $24 \mathrm{~h}$

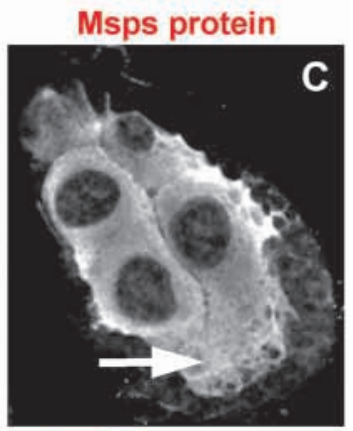

C

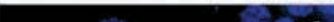

Fig. 6. Overexpression of GFP-PavDEAD stabilises microtubules that have associated Orbit but not Msps protein. Fixed egg chambers expressing GFPPavDEAD (green). DNA is in blue, and tubulin (A,D), Orbit (B,E) and Mini spindle protein $(\mathrm{C}, \mathrm{F})$ in red. (A-F) Extensive regions of colocalisation of tubulin (A) and GFP-PavDEAD are apparent and appear yellow in the merged image (D). Orbit $(\mathrm{B}, \mathrm{E})$ but not Mini spindle protein $(\mathrm{C}, \mathrm{F})$ is found along the microtubules (arrows in B,C,E,F). (G-I) Egg chambers isolated from females fed for 24 hours on unsupplemented yeast paste $(\mathrm{G})$ or yeast paste containing 50 $\mu \mathrm{g} / \mathrm{ml}(\mathrm{H})$ or $1 \mathrm{mg} / \mathrm{ml}$ (I) colchicine. (G) Overexpression of GFP-PavDEAD leads to a cytoplasmic network within the nurse cells (arrow) but not the oocyte (arrowhead). (H) Feeding females $50 \mu \mathrm{g} / \mathrm{ml}$ colchicine for 24 hours results in a partial depolymerisation of the network in the cytoplasm but not at the cortex (arrow). (I) Increasing the colchicine concentration to $1 \mathrm{mg} / \mathrm{ml}$ depolymerises the network almost completely, leaving just a thin ring of filaments around the nurse cell nuclei (arrow). Arrowheads in $\mathrm{H}$ and I point at the oocyte. Bars, $10 \mu \mathrm{m}$ (E,F) and $25 \mu \mathrm{m}(\mathrm{D}, \mathrm{G}-\mathrm{I})$. 
central spindle late in $\mathrm{M}$ phase so as to provide a framework for the assembly of the contractile ring (Adams et al., 1998). The extant pav mutant alleles result in embryonic lethality as a result of failure of cytokinesis in cycle 16, the second cycle after the protein is first required for this process following cellularisation of the embryo. In mitotically dividing cells, Pav-KLP becomes incorporated into the mid-body which is discarded following cytokinesis, whereas in spermatogenesis it is retained in the remnants of the contractile ring that is used to build the ring canals (Carmena et al., 1998). These structures form interconnections between the synchronously developing cells within a common cyst that will ultimately contain 64 spermatids. Here we have shown that Pav-KLP becomes similarly incorporated into the analogous structures that connect the 15 nurse cells and oocyte in the developing egg chamber during oogenesis. Moreover our work defines the central stalk region of the molecule alone as being able to direct this localisation. As ring canals like mid-bodies are derived from the remnants of the cleavage furrow, it is possible we are seeing the end point of a similar series of localisation events to those seen at the end of M-phase. However, as expression of GFP-PavSTALK only becomes maximal after the completion of the mitotic divisions in the germarium, most of the localisation we observe occurs after completion of this process most probably due to incorporation into the growing ring canals. The stalk domains of kinesin-like proteins are known to mediate intermolecular interactions leading to the formation of functional dimeric or trimeric molecules. Consequently, we cannot be certain that association of the stalk with ring canals can occur independently of the remaining portions of the molecule, as we have examined its localisation in flies that are still expressing wild-type protein. Nevertheless, it will be of future interest to search for direct interactions between this domain of the protein and other molecules that could participate in its localisation to these subcellular structures of which actin associated proteins may be among the best candidates because of their abundance in ring canals.

A specific feature of the localisation of Pav-KLP in wild-type oogenesis is its accumulation in the transcriptionally quiescent oocyte nucleus. It is likely that mRNA for the motor protein is actively transported from the nurse cells to the oocyte cytoplasm, where the protein is synthesised for transportation into the nucleus for storage. Subsequently the protein appears to be utilised during female meiosis as it is incorporated into the central spindle pole body
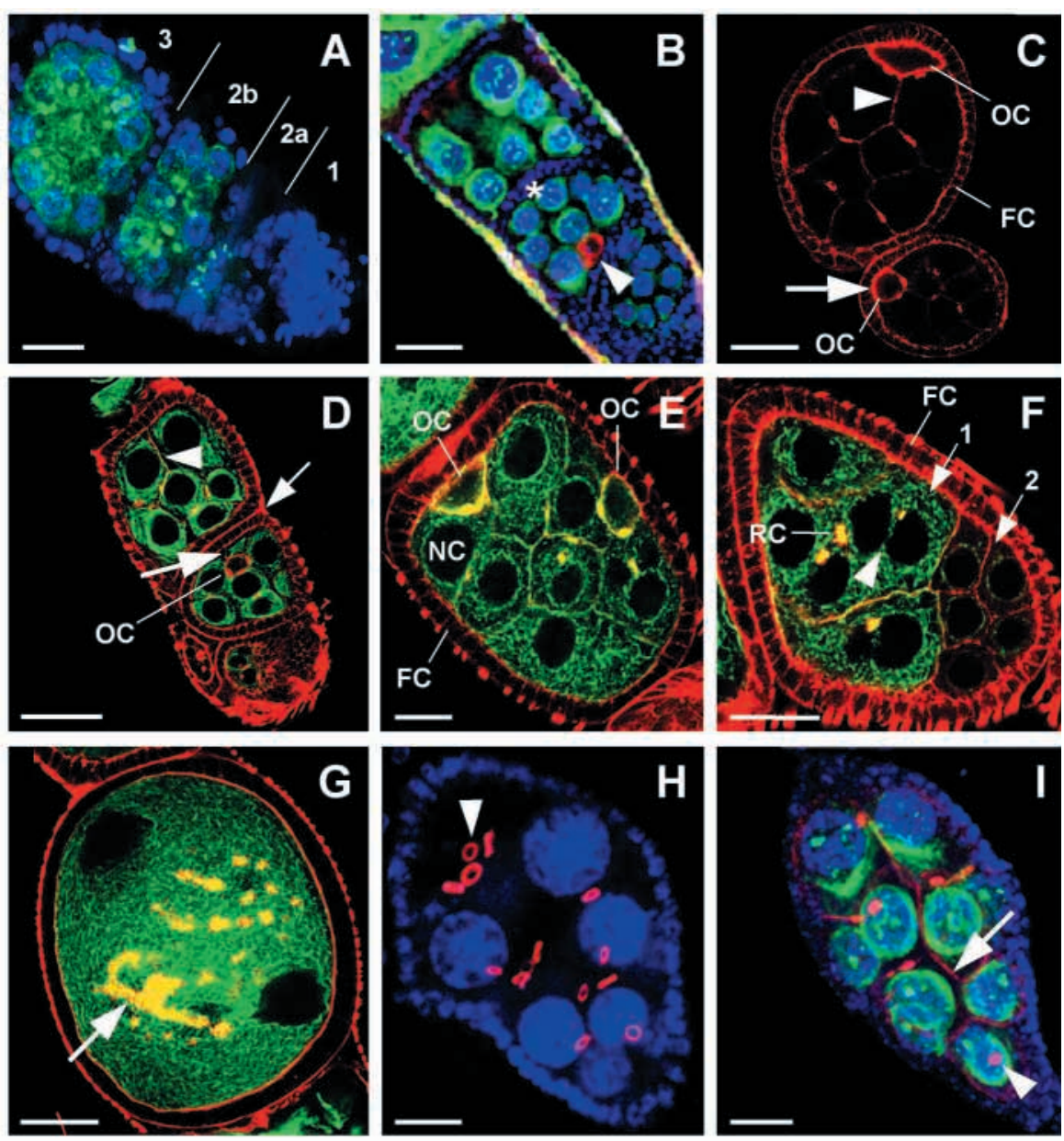

Fig. 7. Overexpression of GFP-PavDEAD results in failure of oocyte growth, loss of interfollicular stalks, fusion of egg chambers and formation of tubulin-actin aggregates. Wild-type $(\mathrm{C}, \mathrm{H})$ and mutant egg chambers expressing either GFP-PavDEAD (B,D,I) or GFP-PavNLS(4-7)* (A,E-G) (green). DNA is in blue and Staufen (B), actin (C-G) and the Hu-li tai shao isoform ADD-60 (H-I) in red. (A) A linear projection of a series of confocal images of a germarium isolated from females expressing GFP-PavNLS(4-7)*. The earliest visible GFP fluorescence appears in ring canals of cysts located in region $2 \mathrm{a}$. The cyst located in region $2 \mathrm{~b}$ contains 16 cells, whereas the stage 1 cyst in region 3 is composed of 32 cells. (B) A young egg chamber with its oocyte localised at the anterior pole (arrowhead). Asterisk, posterior pole. (C) Nurse cells of wild-type egg chamber accumulate actin at their cortex, facilitating the identification of their cell borders (arrowhead). Oocytes (OC) always have four associated ring canals and appear to have a higher concentration of actin at their cortex. They are in direct contact with the follicle cell (FC) layer (arrow). (D) Young mutant egg chambers are not separated by inter-follicular stalks (small arrow). Nurse cells still accumulate actin at their cell borders (arrowhead). The large arrow indicates that the oocyte (OC) is not in contact with the follicular cell layer. (E) A single follicle cell (FC) layer surrounds a 32-cell cyst with two oocytes (OC). All the nurse cells (NC) are of equal size. (F) A single follicle cell layer (FC) surrounds a 32-cell mutant egg chamber with two distinct cysts of 16 cells (1 and 2). Cyst 1 contains some collapsed ring canals (RC) and nurse cells that are not separated by an actin cytoskeleton (arrowhead). (G) An egg chamber in which tubulin-actin aggregates (arrow) are embedded in a cytoplasmic pool of microtubules. Nurse cell nuclei appear as black holes. (H) A wild-type egg chamber in which Hu-li tai shao is present in the ring canals (arrowhead). (I) Hu-li tai shao accumulates in the ring canals (arrowhead) and nurse cell membranes (arrow) of mutant egg chambers. Bars, $10 \mu \mathrm{m}$ (A,E), $20 \mu \mathrm{m}(\mathrm{C}, \mathrm{D}, \mathrm{F}), 50 \mu \mathrm{m}$ (G-I) and $100 \mu \mathrm{m}(\mathrm{B})$. 
that develops between the tandemly arranged spindles of meiosis II (Riparbelli et al., 2000). When expressed at wildtype levels, we cannot see the protein becoming incorporated into the nurse cell nuclei within the egg chamber. It is however, possible that it is simply easier to see the nuclear localisation in the smaller oocyte nucleus. However, we do observe its incorporation into nurse cell nuclei following its overexpression. This is consistent with the known properties of members of the MKLP-1 family, that is following completion of their function in cytokinesis they localise to nuclei during interphase (Nislow et al., 1992; Adams et al., 1998). The only exception is sea urchin $\mathrm{KRP}_{110}$, which seems to have a perinuclear localisation at that stage (Chui et al., 2000).

The overexpression of wild-type Pav-KLP in the female germ line appears not to affect the four rounds of mitosis that take place in the germarium as egg chambers contain the correct number of cells. We believe that oocyte specification may be delayed however, as this is a microtubule-dependent process (Theurkauf et al., 1993). The oocyte is specified but then fails to adopt its correct anterodorsal positioning during stage 2 . It has been argued that the defective oocyte localisation also seen in spindle $C$ mutants is consequential to a delay in oocyte determination (González-Reyes et al., 1997). The mipositioning of the oocyte in egg chambers overexpressing wild-type and mutant forms of Pav-KLP could also be explained as an indirect consequence of a delay to oocyte specification resulting from defects in microtubule-mediated transport. Mispositioning of the oocyte is also seen in mutants for spaghetti squash (sq) (Jordan and Karess, 1997), which encodes a regulatory myosin light chain, which points to the additional possibility of a role for the actin-myosin cytoskeleton in this process.

The major effect resulting from overexpression of GFP-PavKLP is a failure of nurse cells to 'dump' their cytoplasm into the oocyte. In wild-type egg chambers, thick actin filament bundles are required to keep the ring canals free from nurse cell nuclei to enable such a process to occur. Such structures are illuminated by GFP-Pav-KLP expressed at lower levels from the polyubiquitin promoter but are not observed following overexpression of the motor protein. These filaments are also missing in egg chambers of mutants for chickadee, singed and quail (Cant et al., 1994; Cooley et al., 1992; Mahajan-Miklos and Cooley, 1994) where fast cytoplasmic flow is also impaired. Similarly, sqh mutant egg chambers fail to efficiently transport their nurse cell cytoplasm into the oocyte (Edwards and Kiehart, 1996; Wheatley et al., 1995). The overexpression of Pav-KLP may therefore similarly destabilise the actin cytoskeleton, leading to blockage of the ring canals by nurse cell nuclei and the failure of nurse cell 'dumping' that we observe. We suspect that the breakdown of the oocyte/nurse cell membrane and protrusion of nurse cell nuclei into the oocyte cytoplasm may result from an increase in intracellular pressure following the blockage of the ring canals.

Our findings that a set of overlapping nuclear localisation signals are present in the C-terminus of Pav-KLP confirms and extends the study by Deavours and Walker (Deavours and Walker, 1999) who found similar sequences on the mammalian protein. These sequences are not in themselves sufficient to mediate nuclear localisation that can also be prevented by a putative motor inactivating point mutation in the ATP-binding site of the motor protein. We presume that this is due to rigour binding of the putatively immotile protein to microtubules that consequently accumulate in the cytoplasm. It is formally possible that the functional ATP-binding site is a requirement for nuclear import of Pav-KLP, a possibility that could be tested in future by defining and mutating the microtubulebinding site. The rigour-like association of GFP-PavDEAD with cytoplasmic microtubules is, however, reminiscent of that seen with a yeast motor protein, Kar3p, carrying the equivalent G131E point mutation in its ATP-binding site (Meluh and Rose, 1990). Moreover, when a similar mutant of the mitotic centromere-associated kinesin (MCAK) was expressed in $\mathrm{CHO}$ cells it also decorated cytoplasmic microtubules and was excluded from the nucleus (Wordeman et al., 1999), suggesting that this may be a common consequence of inhibiting motor function of kinesin-like proteins.

The microtubule arrays that accumulate when overexpressed Pav-KLP cannot enter the nucleus are more resistant to the microtubule-depolymerising drug colchicine than the microtubule arrays of wild-type egg chambers. Although they retain some specificity for microtubule-associated proteins, shown by their binding of the Orbit but not the Mini spindles protein, it is unlikely that they function normally. Ultimately overexpression of GFP-PavNLS(4-7)* or GFP-PavDEAD leads to a breakdown of the cortical cytoskeleton of the germ line cells. The GFP-tagged Pav-KLP molecules and their associated microtubules appear to colocalise with actin in the cortical cytoplasm, resulting in disruption of cell membranes. This in turn leads to the formation of aggregates enriched in Pav-KLP, tubulin, actin and actin-binding proteins such as $\mathrm{Hu}-$ li tai shao, which is a homologue of adducin that acts as an assembly factor for the spectrin-actin network (Gardner, 1987). It is possibly released from the ring canals as they dissociate and then associates with the actin aggregates. It is not clear whether this effect on the actin cytoskeleton is a direct consequence of the accumulation of microtubules at the cell cortex or whether the ectopic Pav-KLP is sequestering actin regulatory proteins at this site. This might be detrimental when permitted to occur at the cell cortex but may have little effect if restricted to structures such as ring canals, which could occur following overexpression of the stalk domain alone. The breakdown of nurse cell membranes is also caused by mutations in sko, a gene encoding filamin, which cross-links F-actin to nurse cell membranes and ring canals ( $\mathrm{Li}$ et al., 1999). Like the dominant Pav-KLP mutants, sko mutant egg chambers also have nurse cell nuclei that transgress into the oocyte compartment in addition to abnormal ring canals and actin cables (Li et al., 1999). Our findings suggest that accumulated cytoplasmic Pav-KLP leads to a progressively abnormal distribution of actin and its binding proteins and that this is associated with the breakdown of cell borders. This may also facilitate the fusion of egg chambers that can occur at all stages during oogenesis in the dominant mutants.

We will not fully understand the function of Pav-KLP until we know precisely what molecules it interacts with and the nature of the cargoes it carries. As a kinesin-like protein, it might be expected to interact with microtubules as is demonstrated by our studies. Our present findings imply that the protein may also interact with the contractile actin ring or proteins that regulate its function during cytokinesis to become incroporated into the cytokinesis remnant. To date the closest hint of any possible interaction of the protein with any potential 
regulator of the actin cytoskeleton is given by the observation that recruitment of the $C$. elegans MKLP-1 homologue ZEN4 to the spindle midzone requires interaction with a GTPaseactivating protein CYK-4 (Jantsch-Plunger et al., 2000). GTPase-activating proteins of this type regulate the activity of Rho family GTPases, which have a function in contractile ring assembly. Knowledge of the interactions that Pav-KLP makes with other molecules to coordinate microtubule and microfilament behaviour together with the dynamics of these processes will be essential for understanding its roles in mitosis and interphase.

We would like to thank Pernille Rorth for providing the pUASp vector, Daniel Kiehart for providing the anti-Myosin antibody and Daniel St Johnston for the anti-Staufen antibody and the $\alpha 4$ tubulindriven GAL4-VP16 line. We are grateful for a Programme Grant from the Cancer Research Campaign, and to the European Union, which supported Gianluca Minestrini through a Training Network.

\section{References}

Adams, R., Tavares, A., Salzberg, A., Bellen, H. and Glover, D. M. (1998). pavarotti encodes a kinesin-like protein required to organize the central spindle and contractile ring for cytokinesis. Genes Dev. 12, 1483-1494.

Brand, A. H. and Perrimon, N. (1993). Targeted gene expression as a means of altering cell fates and generating dominant phenotypes. Development 118 , 401-415.

Cant, K., Knowles, B., Mooseker, M. and Cooley, L. (1994). Drosophila singed, a fascin homolog, is required for actin bundle formation during oogenesis and bristle extension. J. Cell Biol. 125, 369-380.

Carmena, M., Riparbelli, M., Minestrini, G., Tavares, A., Adams, R., Callaini, G. and Glover, D. M. (1998). Drosophila Polo kinase is required for cytokinesis. J. Cell Biol. 143, 659-671.

Carpenter, A. C. T. (1975). Electron microscopy of meiosis in Drosophila melanogaster females. Structure, arrangement, and temporal change of the synaptonemal complex in wild type. Chromosoma 51, 157-182.

Chou, T. B., Noll, E. and Perrimon, N. (1993). Autosomal p[ovo(D1)] dominant female-sterile insertions in Drosophila and their use in generating germ-line chimeras. Development 119, 1359-1369.

Chui, K., Rogers, G., Kashina, A., Wedaman, K., Sharp, D., Nguyen, D., Wilt, F. and Scholey, J. (2000). Roles of two homotetrameric kinesins in sea urchin embryonic cell division. J. Biol. Chem. 275, 38005-38011.

Cooley, L., Verheyen, E. and Ayers, K. (1992). chickadee encodes a profilin required for intercellular cytoplasm transport during Drosophila oogenesis. Cell 69, 173-184.

Cooley, L. and Theurkauf, W. (1994). Cytoskeletal functions during Drosophila oogenesis. Science 266, 590-595.

Cooley, L. (1998). Drosophila ring canal growth requires Src and Tec kinases. Cell 93, 913-915.

Cullen, C., Deak, P., Glover, D. M. and Ohkura, H. (1999). mini spindles: A gene encoding a conserved microtubule-associated protein required for the integrity of the mitotic spindle in Drosophila. J. Cell Biol. 146, 10051018.

Deavours, B. and Walker, R. (1999). Nuclear localization of C-terminal domains of the kinesin-like protein MKLP-1. Biochem. Biophys. Res. Commun. 260, 605-608.

Edwards, K. and Kiehart, D. (1996). Drosophila nonmuscle myosin II has multiple essential roles in imaginal disc and egg chamber morphogenesis. Development 122, 1499-1511.

Gardner, K. and Bennett, V. (1987). Modulation of spectrin-actin assembly by erythrocyte adducin. Nature 328, 359-362.

Giorgi, F. (1978). Intercellular bridges in ovarian follicles of Drosophila melanogaster. Cell Tissue Res. 186, 413-422.

Gonzalez-Reyes, A., Elliott, H. and St Johnston, D. (1997). Oocyte determination and the origin of polarity in Drosophila: the role of the spindle genes. Development 124, 4927-4937.

Grieder, N., de Cuevas, M. and Spradling, A. C. (2000). The fusome organizes the microtubule network during oocyte differentiation in Drosophila. Development 127, 4253-4264.

Hawkins, N., Thorpe, J. and Schüpbach, T. (1996). encore, a gene required for the regulation of germ line mitosis and oocyte differntiation during Drosophila oogenesis. Development 122, 281-290.

Huynh, J. and St Johnston, D. (2000). The role of BicD, egl, orb and the microtubules in the restriction of meiosis to the Drosophila oocyte. Development 127, 2785-2794.

Inoue, Y., do Carmo Avides, M., Shiraki, M., Deak, P., Yamaguchi, M., Nishimoto, Y., Matsukage, A. and Glover, D. M. (2000). Orbit, a novel microtubule-associated protein essential for mitosis in Drosophila melanogaster. J. Cell Biol. 149, 153-166.

Jantsch-Plunger, V., Gönczy, P., Romano, A., Schnabel, H., Hamill, D., Schnabel, R., Hyman, A. and Glotzer, M. (2000). CYK-4: a Rho family GTPase activating protein (GAP) required for central spindle formation and cytokinesis. J. Cell Biol. 149, 1391-1404.

Jordan, P. and Karess, R. (1997). Myosin light chain-activating phosphorylation sites are required for oogenesis in Drosophila. J. Cell Biol. 139, $1805-1819$.

Kiehart, D. and Feghali, R. (1986). Cytoplasmic myosin from Drosophila melanogaster. J. Cell Biol. 103, 1517-1525.

Koch, E., Smith, P. and King, R. (1976). The division and differentiation of Drosophila cystocytes. J. Morphology 121, 55-70.

Lee, K., Yuan, Y., Kuriyama, R. and Erikson, R. (1995). Plk is an M-phase specific protein kinase and interacts with a kinesin-like protein, CHO1/MKLP-1. Mol. Cell. Biol. 15, 7143-7151.

Li, M., Serr, M., Edwards, K., Ludmann, S., Yamamoto, D., Tilney, L., Field, C. and Hays, T. (1999). Filamin is required for ring canal assembly and actin organization during Drosophila oogenesis. J. Cell Biol. 146, 10611073.

Mahajan-Miklos, S. and Cooley, L. (1994). The villin-like protein encoded by the Drosophila quail gene is required for actin bundle assembly during oogenesis. Cell 78, 291-301.

Matuliene, J. and Kuriyama, R. (1998). The stalk domain is essential for targeting of kinesin-like protein CHO1/MKLP1 to the midzone of mammalian mitotic spindles. Mol. Biol. Cell 9, 243.

Meluh, P. and Rose, M. (1990). kar3, a kinesin-related gene required for yeast nuclear fusion. Cell 60, 1029-1041.

Nislow, C., Lombillo, V., Kuriyama, R. and McIntosh, J. R. (1992). A plus end directed motor enzyme that moves antiparallel microtubules in vitro and localizes to the interzone of mitotic spindles. Nature 359, 543-547.

Powers, J., Bossinger, O., Rose, D., Strome, S. and Saxton, W. (1998) A nematode kinesin required for cleavage furrow advancement. Curr. Biol. 8, 1133-1136.

Raich, W., Moran, A., Rothman, J. and Hardin, J. (1998). Cytokinesis and midzone microtubule organization in Caenorhabditis elegans require the kinesin-like protein ZEN-4. Mol. Biol. Cell 9, 2037-2049.

Riparbelli, M. and Callaini, C. (1995). Cytoskeleton of the Drosophila egg chamber: new observations on microfilament distribution during oocyte growth. Cell Motil. Cytoskeleton 31, 298-306.

Riparbelli, M., Callaini, C. and Glover, D. M. (2000). Failure of pronuclear migration and repeated divisions of polar body nuclei associated with MTOC defects in polo eggs of Drosophila. J. Cell Sci. 113, 3341-3350.

Robinson, D., Cant, K. and Cooley, L. (1994). Morphogenesis of Drosophila ovarian ring canals. Development 120, 2015-2025.

Robinson, D., Smith-Leiker, T., Sokol, N., Hudson, A. and Cooley, L. (1997). Formation of the Drosophila ovarian ring canal inner rim depends on cheerio. Genetics 145, 1063-1072.

Rorth, P. (1998). Gal4 in the Drosophila female germline. Mechanisms of Development 78, 113-118.

Sadowski, I., Ma, J., Triezenberg, S. and Ptashne, M. (1988). GAL4-VP16 is an unusually potent transcriptional activator. Nature 335, 563-564.

Sellitto, C. and Kuriyama, R. (1988). Distribution of a matrix component of the midbody during the cell cycle in Chinese Hamster Ovary cells. J. Cell Biol. 106, 431-439.

Severson, A., Hamill, D., Carter, J., Schumacher, J. and Bowerman, B. (2000). The Aurora-related kinase AIR-2 recruits ZEN-4/CeMKLP1 to the mitotic spindle at metaphase and is required for cytokinesis. Curr. Biol. 10, 1162-1171.

St Johnston, D., Beuchle, D. and Nüsslein-Volhard, C. (1991). staufen, a gene required to localize maternal RNAs in the Drosophila egg. Cell 66, 51-63.

St Johnston, D. (1995). The intracellular localization of messenger RNAs. Cell 81, 161-170.

Theurkauf, W., Smiley, S., Wong, M. and Alberts, B. (1992). Reorganization of the cytoskeleton during Drosophila oogenesis: Implications for axis specification and intercellular transport. Development 115, 923-936.

Theurkauf, W., Alberts, B., Jan, Y. and Jongens, T. (1993). A central role 


\section{6}

\section{Journal of Cell Science 115 (4)}

for microtubules in the differentiation of Drosophila oocytes. Development 118, 1169-1180.

Theurkauf, W. (1994). Microtubules and cytoplasm organization during Drosophila oogenesis. Dev. Biol. 165, 352-360.

Tilney, L., Tilney, M. and Guild, G. (1996). Formation of actin filament bundles in the ring canals of developing Drosophila follicles. J. Cell Biol. 133, 61-74.

Verheyen, E. and Cooley, L. (1994). Looking at oogenesis. Methods Cell Biology 44, 545-561.

Warn, R., Gutzeit, H., Smith, L. and Warn, A. (1985). F-actin rings are associated with the ring canals of the Drosophila egg chambers. Exp. Cell Res. 157, 355-363.

Wheatley, S., Kulkarni, R. and Karess, R. (1995). Drosophila nonmuscle myosin II is required for rapid cytoplasmic transport during oogenesis and for axial nuclear migration in early embryos. Development 121, 19371946.
Wordeman, L., Wagenbach, M. and Maney, T. (1999). Mutations in the ATP-binding domain affect the subcellular distribution of mitotic centromere-associated kinesin (MCAK). Cell Biol. Int. 23, 275-286.

Yue, L. and Spradling, A. C. (1992). hu-li tai shao, a gene required for ring canal formation during Drosophila oogenesis, encodes a homolog of adducin. Genes Dev. 6, 2443-2454.

Zaccai, M. and Lipshitz, H. (1996a). Differential distributions of two adducin-like protein isoforms in the Drosophila ovary and early embryo. Zygote 4, 159-166.

Zaccai, M. and Lipshitz, H. (1996b). Role of Adducin-like (hu-li tai shao) mRNA and protein localization in regulating cytoskeletal structure and function during Drosophila oogenesis and early embryogenesis. Dev. Genet. 19, 249-257.

Zernicka-Goetz, M., Pines, J., Ryan, K., Siemering, K., Haseloff, J., Evans, M. and Gurdon, J. (1996). An indelible lineage marker for Xenopus using a mutated green fluorescent protein. Development 122, 3719-3724. 